\title{
Different uses of Bronfenbrenner's ecological theory in public mental health research: what is their value for guiding public mental health policy and practice?
}

\author{
Malin Eriksson ${ }^{1} \cdot$ Mehdi Ghazinour ${ }^{2}$. \\ Anne Hammarström ${ }^{3}$
}

\begin{abstract}
Bronfenbrenner's ecological theory is appealing as a conceptual tool for guiding public mental health interventions. However, his theory underwent significant changes since its first inception during the late 1970s until his death in 2005 , due to which the implications that can be drawn might differ depending on what concepts (i.e. early or later) of the theory is utilized. The aim of this paper was to examine how different concepts of Bronfenbrenner's theory have been utilized in (public) mental health research, and to analyse the value of these different uses for guiding public mental health policy and practice. A systematic search for articles that have utilized concepts of Bronfenbrenner's theory within the field of mental health resulted in a review of 16 published papers. We found that one set of papers $(N=10)$ used the early concepts of ecological systems without investigating interactions between these systems, while another set of papers used the concepts of ecological systems by also investigating interactions within and between these systems $(N=4)$. Another limited set of papers $(N=2)$ utilized the later concepts of proximal processes and the PPCT model. Our results show that studies using Bronfenbrenner's ecological system concepts by clearly considering interactions
\end{abstract}

Electronic supplementary material The online version of this article (https://doi.org/10.1057/s41285018-0065-6) contains supplementary material, which is available to authorized users.

Malin Eriksson

Malin.eriksson@umu.se

Mehdi Ghazinour

Mehdi.ghazinour@umu.se

Anne Hammarström

Anne.hammarstrom@pubcare.uu.se

1 Department of Social Work, Umeå University, 90187 Umeå, Sweden

2 Police Education Unit at Umeå University, Umeå, Sweden

3 Department of Public Health and Caring Sciences, Uppsala University, Uppsala, Sweden 
between and within these systems can result in recommendations that are most useful for guiding public mental health policy and practice.

Keywords Bronfenbrenner · Ecological theory · Mental health $\cdot$ Public mental health interventions $\cdot$ Review

\section{Introduction}

Mental health is an integral part of health, defined as "a state of well-being in which an individual realizes his or her own abilities, can cope with the normal stresses of life, can work productively and is able to make a contribution to his or her community" (WHO 2014). Thus, mental health is more than just the absence of mental disorders or disabilities but a fundamental for good quality of life (WHO 2012). Mental illness is a growing global public health problem. The burden of mental and substance use disorders increased by $37.6 \%$ between 1990 and 2010 (Whiteford et al. 2013). In 2010, mental and substance abuse disorder accounted for $7.4 \%$ of disability-adjusted life years (DALYs) worldwide, not the least caused by depressive and anxiety disorders (Whiteford et al. 2013). Depression alone accounts for $4.3 \%$ of the global burden of disease and is among the largest single causes of disability worldwide, particularly for women (WHO 2013). A review of the global burden of mental disorders (Kessler et al. 2007), based on data from the WHO mental health survey in 28 countries around the globe, concludes that mental disorders commonly occur in the general population worldwide, often making a debut at an early age, and are often associated with significant adverse costs to society. Since many mental disorders begin in childhood and adolescence (Kessler et al. 2007), early detection and interventions are needed. Given the magnitude of mental health problems worldwide, improvements in population health are only possible if countries make prevention of mental health disorders a public health priority (Whiteford et al. 2013).

Determinants of mental health and illness include individual, social and societal factors, and their interaction with each other (Sturgeon 2007). Thus, mental health needs to be understood from biological, psychological as well as sociocultural perspectives (Kendler 2008), and in order to prevent mental illness and promote mental health, there is a need to simultaneously target several multilayered factors (WHO 2012). Consequently, a broad public health perspective is needed to promote mental health and prevent mental illness (WHO 2005). Public mental health promotion focuses on the social determinants of health in order to strive for positive mental health for all (Jané-Llopis et al. 2005). The need for a holistic approach in (mental) health promotion and intervention has been underlined in several international health documents, not the least in the Alma Ata Declaration (WHO 1978), the Ottawa Charter (WHO 1986) and later by the WHO Commission on the Social Determinants of Health (CSDH, WHO 2008). However, in order to clearly understand and act upon these multilayered and interacting social and biological processes that determine mental health, theory is crucial. Theory offers understandings of the causal pathways between various factors and health and disease, 
and can thus guide the planning and design of public mental health interventions. Despite this, the use of theory in epidemiology and public health research and interventions is still quite sparse (Krieger 2001). Further, despite the renewed interest in the social determinants of health, the dominant theories in epidemiology and public health have so far mainly been biomedical or lifestyle oriented, implying a focus on individual-level exposures, behaviours and interventions (Krieger 2014). There is clearly a need for theories embracing the complex and multifaceted pathways in mental health, in order to be useful for guiding public mental health policy and practice.

\section{An ecological approach to public mental health}

An ecological perspective offers a way to simultaneously emphasize both individual and contextual systems and the interdependent relations between these two systems, and thus offers a variety of conceptual and methodological tools for organizing and evaluating health-promotion interventions (Stokols 1996). From a public (mental) health perspective, ecological thinking is appealing since it encompasses several contexts in a very broad sense, including trends such as globalization, urbanization and environmental change, together with (but not solely focusing on) attributes and behaviours of individuals-all relevant aspects for understanding and determining public health (McLaren and Hawe 2004). Ecological theories emanate from many disciplines, but health research has mainly been influenced by psychology, including community and developmental psychology (Richard et al. 2011). The developmental psychologist, Urie Bronfenbrenner, stands out as one of the most influential contributors to ecological thinking in health research. Influenced by his mentor, Kurt Lewin, Bronfenbrenner (1977) started to develop his ecological theory as a new theoretical perspective for understanding human development. His theory underwent significant changes since its first inception during the late 1970s, as he constantly revised the theory until his death in 2005. Even though Bronfenbrenner developed his theory to understand human development, it has been extensively applied in many other fields including health research (see e.g. Richard et al. 2011; Grzywacz and Fuqua 2000).

The evolution of Bronfenbrenner's theory has been described in different phases (Rosa and Tudge 2013): from an ecological approach to human development during the initial phase (1973-1979), followed by a stronger emphasis on the role of the individual and developmental processes during 1980-1993. Finally, in the last phase (1993-2006), the Process-Person-Context-Time model (PPCT) was developed and described as the most appropriate research design for the theory. This development of Bronfenbrenner's theory has, however, been neglected in most studies. Tudge et al. (2009) examined 25 papers, all explicitly claiming to be based on Bronfenbrenner's theory and published in 2001 or later, and found that only four of these studies built on the latest form (PPCT) of the theory. In this paper, we use the term "Bronfenbrenner's theory" when referring to any of the versions of his theory, and elsewhere we specify what version or concepts we refer to.

Bronfenbrenner's theory is clearly appealing as a conceptual tool for guiding interventions within the field of public mental health. However, the implications that 
can be drawn for public mental health policy and practice might differ depending on what concepts (i.e. early or later) of the theory are utilized, and how these concepts are applied. Therefore, the aim of this paper was to examine how different concepts of Bronfenbrenner's theory have been utilized in (public) mental health research, and to analyse the value of these different uses for guiding public mental health policy and practice.

This implies that we do not intend to judge what version of the theory is the most correct to use, but rather to assess the value of using different concepts of the theory for guiding public mental health interventions. Even though Bronfenbrenner himself acknowledged the latest form of his theory as the most appropriate (Bronfenbrenner and Evans 2000), we adhere to a pragmatic view of knowledge and theory. In line with Bryant (2009), we believe that "knowledge exists in the form of statements or theories which are best seen as instruments or tools; coping mechanisms, not onceand-for-all-time truths. ... Rather knowledge [or theory, our note] is a web or a network of statements rather than an edifice, and the value of any form of knowledge [or theory, our note] is its usefulness and applicability which may be constrained in terms of time and place and user" (Bryant 2009, pp. 4-5).

Thus, we believe that even use of earlier concepts from Bronfenbrenner's ecological theory might potentially be useful for guiding public mental health interventions.

\section{Methodological approach}

Our overall research approach was theoretical in that we examined how different concepts (i.e. earlier versus later) of Bronfenbrenner's theory have been used within the public mental health field and analysed the value of these uses for guiding public mental health policy and practice. The study was conducted in several distinct steps. Initially, we systematically read through a selection of Bronfenbrenner's key publications (starting with earlier publications and stepwise continuing with later publications) in order to get a good overview and understanding of how his theory evolved and developed over time. Next, we identified key concepts and basic assumptions in the early and later versions of his theory that could be contrasted and compared with regard to mental health.

After that, we systematically searched for published articles that have utilized Bronfenbrenner's theory within the field of mental health. This search was conducted to identify illustrative examples of how different concepts of his theory have been applied in mental health research. We searched for articles in the database Web of Sciences, using the following search terms: "Bronfenbrenner" AND "mental health" (topic, all years until November 9, 2015). This search resulted in 34 articles.

These 34 articles were briefly read through to assess their relevance for the purpose of our study. Our criterion for selecting articles for further review was that it should be possible to identify from the article what concepts of Bronfenbrenner's theory were utilized (i.e. earlier or later concepts), even if not specifically stated by the authors. We made an independent assessment of what concepts of the theory 
were utilized in each paper, beyond the references used by the authors themselves. In some cases, the authors had referred, for example, to Bronfenbrenner's later texts, without using concepts of later versions of the theory. Other inclusion criteria were that the concepts used should have been clearly described/defined and applied in the study (as opposed to only discussed in relation to results). Further, some kind of mental health indicator ought to have been used as an "outcome variable". Articles that did not fulfil these criteria were excluded from further analysis, including purely methodological and/or theoretical papers. In this way, 15 of the 34 papers were selected for further analysis. In addition, another relevant article was found in the database Pub-Med, using "Bronfenbrenner" as the search term (all fields, until 9 November 2015).

In total, 16 relevant articles were identified, and these papers were used as a basis for analysing the value of using different concepts of Bronfenbrenner's theory for guiding public mental health policy and practice. The selected articles were summarized by content in Appendix 1. They are included in the list of references (indicated by *) and are cited below.

\section{Analysis of selected articles}

All 16 papers were read and reread thoroughly in order to identify how concepts of Bronfenbrenner's theory were utilized; the aims of using his theory within the field of mental health; study design; ecological concepts used; main findings with regard to mental health; conclusions drawn and implications for public mental health interventions (see Appendix 1 for a summary of this review). This review was then used as a basis for analysing the overall strengths and limitations of using different concepts of the theory with regard to guiding public mental health interventions.

\section{Results and reflections}

This section is structured in three parts: first, we briefly present the development of Bronfenbrenner's theory over time and compare the analytical focus between different conceptual versions of his theory, with regard to mental health. Next, we present a summary of how various concepts of Bronfenbrenner's theory have been applied in mental health research, and finally we discuss the value of these different uses of the theory for guiding public mental health interventions.

\section{Key concepts and basic assumptions in early and later versions of Bronfenbrenner's theory}

In this section, we give a brief overview of the development of Bronfenbrenner's theory during the period 1973-2006, mainly based on the three phases proposed by Rosa and Tudge (2013). 


\section{Phase 1 (1973-1979)—an ecological approach to human development}

During the 1970s, Bronfenbrenner named his emerging theory an "ecological model of human development" (Rosa and Tudge 2013). Ecology was defined as a fit between the individual and his/her environment. In order to develop, and not only survive, the fit between the individual and its environment must be even closer (Bronfenbrenner 1975). In this earliest stage of the theory, Bronfenbrenner described the ecological environment as composed of systems at four different levels. The microsystem contains relations between the individual and the immediate environment surrounding the individual, such as the home, school and workplace (Bronfenbrenner 1977). The mesosystem comprises interrelations between major settings containing an individual, such as relations between home and school, home and peer-groups, etc. (Bronfenbrenner 1977). The exosystem embraces social structures-major institutions of the society-such as the world of work, the mass media and public agencies. These social structures do not themselves contain the developing person but impinge upon the immediate settings in which that person is found, and as such influence what is going on in these settings (Bronfenbrenner 1977). The macrosystem consists of the blueprints of a particular society such as laws and regulations but also unprinted rules and norms (Bronfenbrenner 1978). Analysing the composition of these ecological systems as well as interactions between and within these systems and individual factors was regarded as crucial in order to understand and explain a developmental outcome. The requirement for ecological research was to include at least two different ecological systems in the analysis to understand a particular developmental outcome (Bronfenbrenner 1975). In addition, Bronfenbrenner also emphasized ecological transitions in his early texts, i.e. shifts from one ecological context to another that every person undergoes throughout life (Bronfenbrenner 1979), such as starting school, getting a sibling, marriage, divorce, getting a new teacher, moving, etc. Investigating the characteristics, qualities and impact of the ecological transitions an individual goes through was also proposed by Bronfenbrenner (1978) as an important part of ecological research.

\section{Phase 2 (1980-mid-1990s)_adding biology and chronosystem into the ecological framework}

During this period, Bronfenbrenner further developed ideas about how individual characteristics interplay with context. In a paper from 1994 about the relation between nature and nurture, Bronfenbrenner and Ceci state that genetic material is not finished traits, but interacts with environmental experiences in determining developmental outcomes. According to them (Bronfenbrenner and Ceci 1994) human development involves interaction between the biological and psychological person and his/her environments, and the realization of human potential requires an intervening mechanism that connects the inner with the outer in a two-way process occurring over time.

During this phase, Bronfenbrenner put more emphasis on the close and reciprocal face-to-face interactions with the child's immediate environment (Bronfenbrenner 
and Ceci 1994). This was later referred to as "proximal processes"-a concept that was fully developed in phase 3 (see below). During this phase, Bronfenbrenner also developed his thinking about time by adding "chronosystems" to his ecological model. Although Bronfenbrenner mentioned time already in his book from 1979, the concept of chronosystem was not added until this second phase. By adding chronosystems, Bronfenbrenner wanted to take into account changes over time, not only within the person but also in the environments in which that person is found, to investigate how these changes may affect a person's developmental outcomes (Bronfenbrenner 1986). This could entail investigating how changes in a parent's work status (part-time, full-time, etc.) over time during a child's school ages could affect patterns of parent-child communication, and how these patterns in turn could influence the child's achievement and social behaviour in school (Bronfenbrenner 1986).

\section{Phase 3 (mid-1990s-2006)—a Process-Person-Context-Time (PPCT) model}

During this final phase, Bronfenbrenner finalized his theory by developing his thinking about "proximal processes", now referred to as the "engine of development". Proximal processes involved reciprocal interaction between the developing individual and other (significant) persons, objects and symbols in his/her immediate environment, and these processes could involve activities between parents and child and child and child, such as playing, reading and learning new skills (Bronfenbrenner 1995). Proximal processes were viewed as the most powerful predictor of human development and Bronfenbrenner wanted to show how individual characteristics, together with aspects of the environment, influence proximal processes (Rosa and Tudge 2013). In specifying the nature, operation and developmental effects of proximal processes, Bronfenbrenner "re-conceptualized" the microsystem. According to him, proximal processes operate within microsystems and involve interaction with three features of the immediate environment: persons, objects and symbols. Persons were further referred to as "significant others" by adopting Mead's terminology (Bronfenbrenner 1995). In further trying to rule out why different developmental outcomes vary between individuals, Bronfenbrenner and his colleagues (Bronfenbrenner and Ceci 1994; Bronfenbrenner 1995; Bronfenbrenner and Evans 2000) developed this hypothesis into a ProcessPerson-Context-Time model (PPCT), and the model was developed to guide how bioecological research best could be conducted (Rosa and Tudge 2013). Considering Process would imply assessment of regularly occurring activities and interactions with significant persons, objects and symbols in the developing individual's lives. Accounting for Person would require analysing how individual characteristics influence proximal processes, such as assessing how age, gender, temperament, intelligence, etc. influence these activities and interactions. Context was described as involving four interrelated systems: microsystem (the immediate environment where the developing person engages in activities and interactions, i.e. where proximal processes occur), mesosystem (interrelations among several microsystems in which that person is situated), exosystems (contexts having an indirect influence on the person) and finally, macrosystem (contexts with a shared 
belief system). Adding Context could thus imply evaluating the influences of different exosystems (such as parent's work or the mass media) and/or different macrosystems (such as values within cultural groups) on the proximal processes of interest. Finally, considering aspects of Time would ideally require a longitudinal study with at least two measurement points taking into account the current point of historical time (Tudge et al. 2009). Bronfenbrenner never implied that all four elements have to be included in every study, but underlined that studies involving the PPCT model should focus on proximal processes, showing how they are influenced both by characteristics of the developing individual and by the context in which they occur (Tudge et al. 2009).

Table 1 shows that the core of analysis of mental health studies applying the earliest concepts (developed in phase 1) of Bronfenbrenner's theory would be to examine how mental health is determined by mutual influence between individual factors and the ecological systems surrounding an individual/group, as well as interactions between and within these ecological systems. Further, mental health studies applying later concepts (from phase 2) of Bronfenbrenner's theory would also add chronosystem to the ecology. Finally, studies using the most mature concepts of the theory (developed in phase 3) would focus on proximal processes and applying the PPCT model. As Table 1 shows, it is also clear that the earlier phase of the theory put more emphasis on context, while the later phases put more emphasis on the closer environment.

\section{Different uses of Bronfenbrenner's theory in mental health research}

From the 16 reviewed articles, we were unable to identify articles that could be regarded as "purely" using concepts from just one of the identified phases of the theory, as outlined by Rosa and Tudge (2013). This probably reflects a general unawareness of how Bronfenbrenner's theory developed over time, a fact also noted by others (Tudge et al. 2009; 2016). Instead, we found three main ways of using concepts from Bronfenbrenner's theory within our 16 reviewed papers. One set of papers $(N=10)$ used the concepts of ecological system (of which five also included chronosystem) without investigating interactions between these systems, while another set of papers used the concepts of ecological systems by also investigating interactions within and between these systems $(N=4)$. Another limited set of papers $(N=2)$ utilized the later concepts of proximal processes and the PPCT model. Two of the reviewed articles (Mutumba and Harper 2015; Romano et al. 2015) used concepts of Bronfenbrenner's theory (at least partly) in conjunction with other theoretical frameworks, while the others were based solely on concepts from Bronfenbrenner's theory. Table 2 summarizes how the theory has been utilized within these three identified groups of articles with regard to the purpose of using Bronfenbrenner's theory; study designs; concepts utilized; main results; implications for public mental health policy and interventions; and strengths and weaknesses for guiding public mental health policy and practice. 


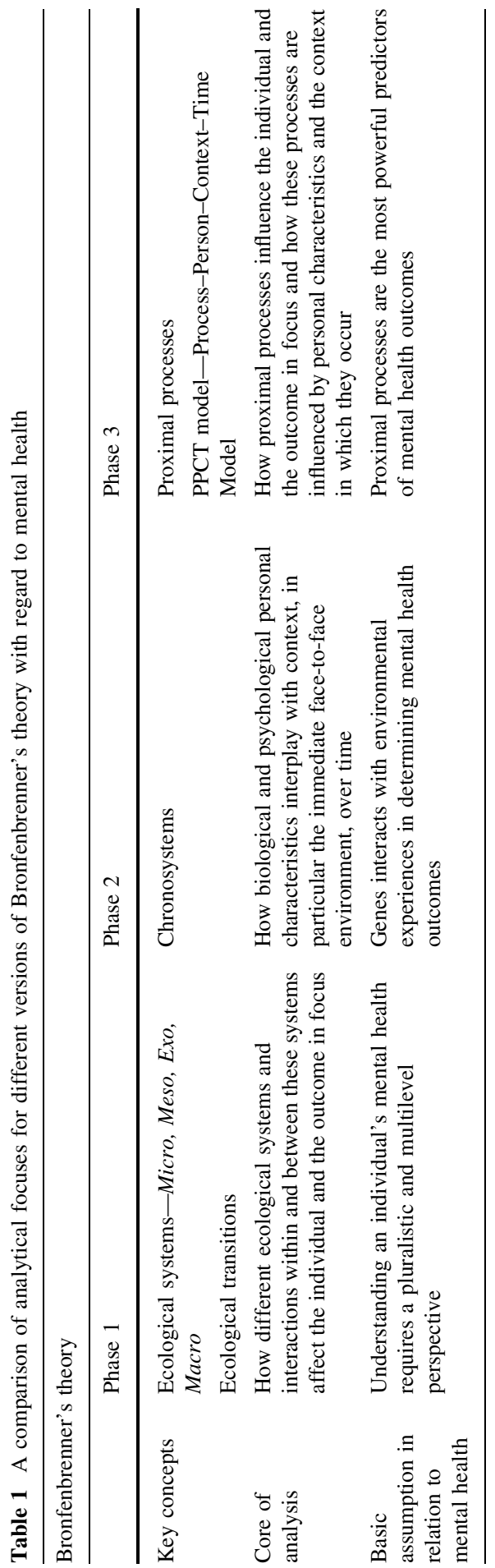




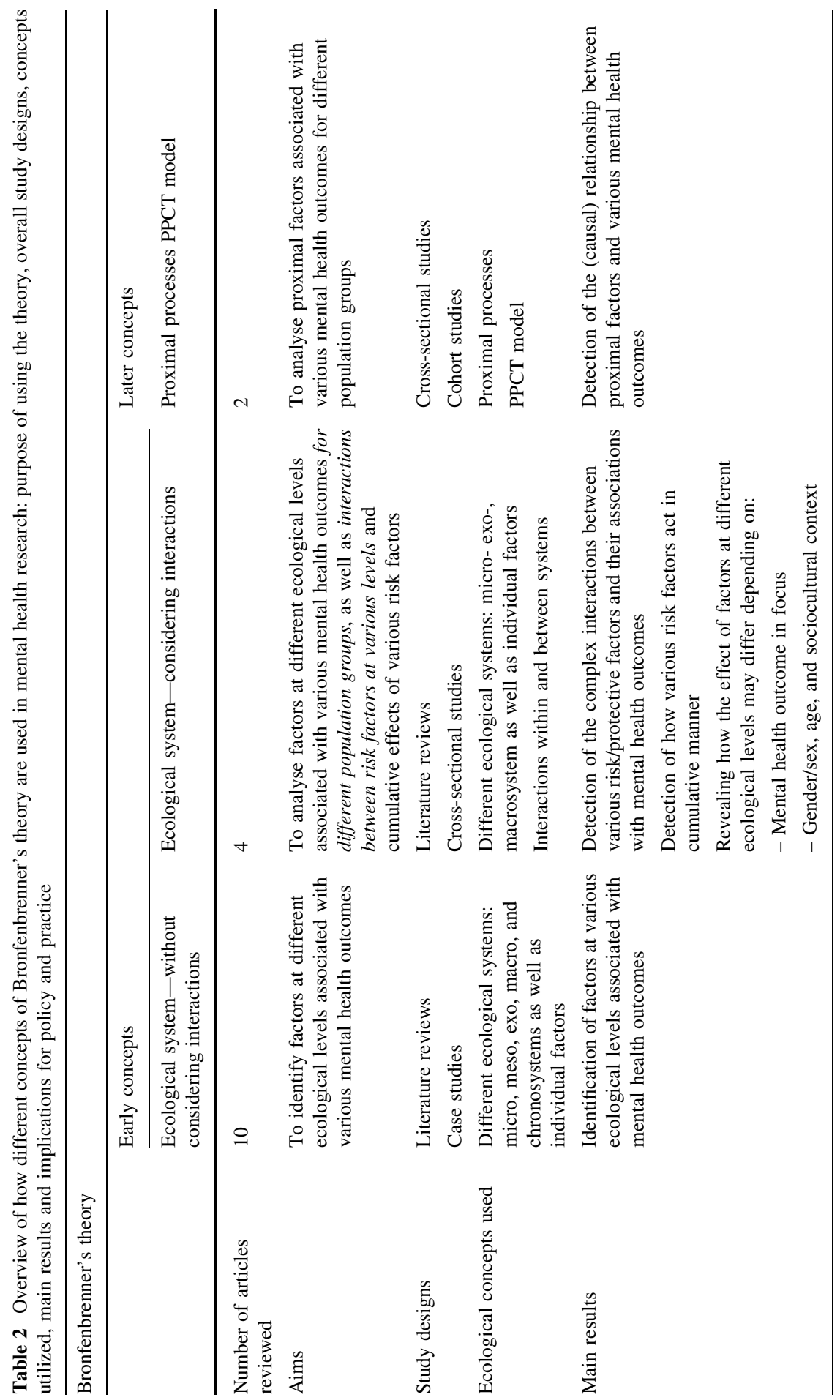




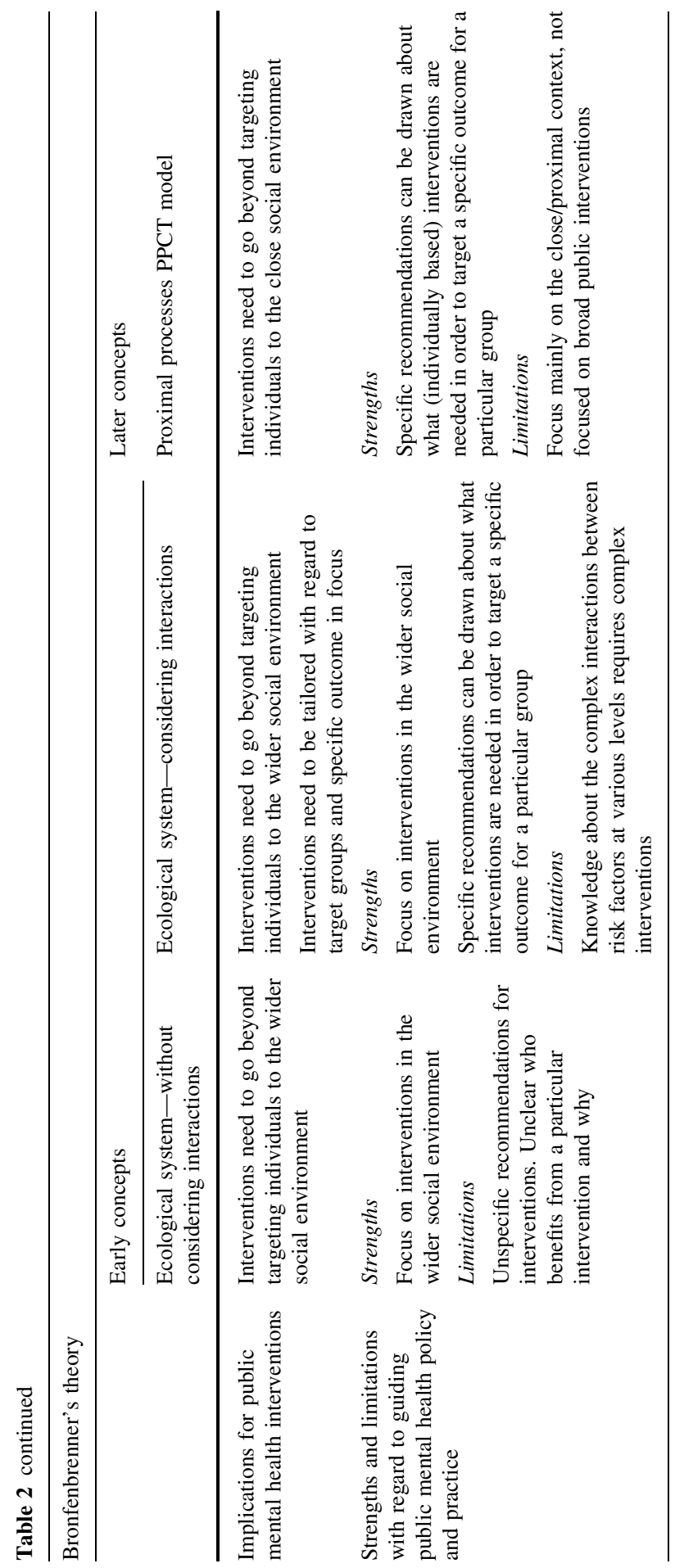


Studies utilizing ecological systems concepts without considering interactions between and within ecological systems

Table 2 illustrates that ten out of 16 reviewed articles utilized ecological systems concepts without clearly considering interactions within and between these different ecological systems. This implies that the majority of our reviewed articles utilize Bronfenbrenner's theory in a way that was never intended by Bronfenbrenner himself, since even in his earliest writings he underlined the importance of considering interactions within and between ecological systems (Bronfenbrenner 1975). These ten studies have in common that they aim to go beyond individual risk factors for understanding various mental health outcomes, since previous studies have mainly focused on personal characteristics without considering the larger surrounding environments. Thus, these studies use concepts of Bronfenbrenner's ecological theory for identifying factors at different ecological levels that can explain the development of mental health outcomes in general (Pilgrim and Blum 2012; Aston 2014), but also more specific mental health related outcomes such as parenting capacity (Grant and Guerin 2014), bullying and peer victimization in schools (Hong and Espelage 2012; Huang et al. 2013; Upton Patton et al. 2013), school shootings (Hong et al. 2010), and sexual assaults (Campbell et al. 2009).

The concepts used in these studies are naturally different ecological systems (micro, meso, exo, macro, and chrono) as well as various individual factors. Consequently, the results from these studies end up identifying factors at different ecological levels that are positively and/or negatively associated with the particular mental health outcome in focus. Further, even if the need to consider interactions between and within ecological systems in order to understand mental health outcomes is pointed out in (some of) these studies, this is not explicitly done in the analyses. As an example, Hong and Espelage (2012) in their literature review identified risk factors at all ecological levels associated with bullying and peer victimization in school, but did not really consider interactions between these different systems beyond bringing up the fact that the associations between parentyouth relationships and bullying may differ for boys and girls. Likewise, Campbell et al. (2009) point out that the "next step" of developing a model of rape recovery would be to examine interactions across different levels of the social ecology, in order to get a comprehensive understanding. They (Campbell et al. 2009) further discuss that the mixed results found in their review regarding the influence of individual characteristics and assault characteristics on the mental health effects of sexual assaults probably are due to unexplored cross-level interactions.

The policy implications that can be drawn from these studies are consequently quite unspecific. When discussing the policy implications from their review of factors associated with school bullying and peer victimization in the People's Republic of China, Huang et al. (2013) end up in general recommendations such as the need for (1) considering individual factors (age and gender) by targeting younger children and boys in particular (since these groups are more prone to engage in bullying); (2) setting up parent education for abusive parents (micro level); and (3) restricting children's exposure to media violence (exo level). Similarly, Hong et al. (2010), when discussing the policy implications of how to 
prevent school shootings, end up with unspecific recommendations such as the need for skill-building programmes for parents and youths on communication and conflict resolution (micro level); setting up of arenas where parents and teachers can meet (meso level); provision of educational material about the detrimental effects of exposure to media violence (exo level); implementing school programmes that address gun violence in school (macro level); and educating governments about the relation between social conditions and negative outcomes among immigrants (chrono level). Likewise, Yakushko and Chronister (2005) outline various counselling strategies and interventions at different ecological levels for immigrant women in the US. They suggest the importance of the counsellor valuing immigrant women's cultural experiences (individual level); assessing changes in women's family structure (micro level); strengthening existing support networks (meso and exo levels); and informing about laws that prohibit discrimination (macro level). Although these recommendations are relevant and valid, one might assume that these recommendations could have been brought up even without using an ecological theoretical framework. Likewise, Mutumba and Harper (2015) use an ecological framework to identify the risk and protective factors for mental health diseases for sexual minority youth at different ecological levels. However, in their recommendations for treatment and support, they end up in very broad recommendation such as "developing and enforcing child protection systems", without even linking these recommendations to the ecological levels where they "belong".

Thus, even though these studies bring up general suggestions for how to move beyond individual factors to also intervene in the social environment, they do not give any detailed advice on how to prevent a specific mental health outcome for a particular target group. One exception though is Pilgrim and Blum's (2012) study about the risk and protective factors for adolescents' mental and physical health in the English-speaking Caribbean. They identified that girls are more likely to experience internalizing problems, while boys are more likely to have externalizing problems. Therefore, interventions focusing on skills training for emotional regulations, coping skills for managing stress and dietary behaviour may be especially beneficial for girls, while policies advocating for reduced youth access to drugs and weapons and programmes focusing on conflict resolution skills may be especially beneficial for boys. However, beyond this example, studies utilizing early concepts of Bronfenbrenner's theory without considering interactions between and within ecological systems tend just to include many factors at various levels in a mental health-risk model, without being able to rule out the complex interactions between these factors. These kind of results easily lead to the conclusion that "everything affects everything", which is not very helpful for health policy and planning (Grzywacz and Fuqua 2000).

\section{Studies utilizing ecological systems concepts by considering interactions} within and between systems

Four of the reviewed articles used a more multifaceted ecological analysis by taking into account interactions within and between ecological systems. Beyond identifying factors within different ecological systems (micro, meso, exo and macro) 
associated with various health outcomes for different groups of people (e.g. based on gender, age, etc.), these articles also aim to analyse interactions between risk factors at different levels and if and how risk factors act in a cumulative manner. Thus, these studies move beyond focusing on isolated variables and contribute to an understanding of the complex interactions between various risk and/or protective factors and their effect on mental health outcomes for different groups of people.

When analysing risk factors for problem behaviour among English and Indian children living in London, Atzaba-Poria et al. (2004) not only identified risk factors at different ecological levels, but also analysed how much of the risk could be attributed to each of the different ecological levels, as well as cumulative risks of various exposures. They found that regardless of the specific type of risk, the more accumulated risks children experienced, the higher the levels of total problem behaviour. They were also able to detect how different kinds of accumulated risks (emanating from the micro, meso or exo level or individual factors) were associated with different behavioural problems (aggressive behaviour versus anxiety and depression). Likewise, Behnke et al. (2011) were able to detect how the association between factors at different ecological levels and depressive symptoms differed for girls and boys. Equally, Romano et al. (2015), in their review of the complex relationship between childhood maltreatment and later academic achievement and mental health, found that the negative consequences of childhood maltreatment seemed to be greater for boys than girls. They also found that some forms of maltreatment (early in life, multiple, neglect) seemed to be especially harmful for academic achievements. Further, McDaniel et al. (2012) explored interactions between micro and meso level interactions and found that blogging (meso level interactions) positively influenced family relations (micro level interactions) which in turn had a positive effect on maternal well-being. Thus, the positive effects of the mesosystem went through interactions with the microsystem.

The results of these studies show how the influence of different risk factors may vary for different groups and depending on the mental health outcome in focus. Thus, the recommendations for interventions that can be drawn from these studies are in general more specific. One clear example is the study by Atzaba-Poria et al. (2004). They found that interventions within the microsystem were needed in order to prevent aggressive behaviours among children, while interventions in the exosystems (peer and parental relations) were needed in order to prevent anxious and depressive behaviours among children. Behnke et al.'s study (2011) further suggests that interventions targeting adolescents' self-esteem and depressive symptoms need to be tailored differently for boys and girls; targeting neighbourhood factors might have to be especially tailored to meet the needs of boys while targeting societal discrimination has to specifically address the needs of girls. Finally, the review by Romano et al. (2015) suggests that some forms of child maltreatment—neglect, early and multiple_-might be especially important to detect and intervene against in order to promote later academic achievement and mental health. These recommendations can thus be used for tailoring interventions for the specific target group and outcome in focus. Consequently, studies using Bronfenbrenner's ecological system concepts by clearly considering interactions between and within these systems can result in recommendations that are most useful for 
guiding public mental health policy and practice. However, even if these recommendations might be specific, one needs to acknowledge that the recommendations might not be too easy to implement in practice since they require quite complex societal interventions.

\section{Studies applying later concepts of the theory}

We identified only two studies that have utilized the later concepts of Bronfenbrenner's theory. Our review suggests, in line with others (Tudge et al. 2009; Tudge et al. 2016), that the later version of Bronfenbrenner's theory is still less utilized in research, including the field of public mental health. Liem et al. (2010) used longitudinal data from a random sample of young people in Boston, USA, to explore differences in mental health outcomes (depressive symptoms, life satisfaction) between high school dropouts and graduates, while Williams and Nelson-Gardell (2012) used data from the US National Survey of Child and Adolescent Well-Being to examine factors predicting resilience in sexually abused adolescents. Both these studies used all or some elements from the PPCT model to analyse factors positively and negatively associated with mental health outcomes for different population groups. In these studies, proximal factors are given more "weight" for understanding mental health outcomes, although especially Williams and Nelson-Gardell (2012) also considered some more distal factors (family SES) that proved to be of equal importance in predicting clinical symptoms in sexually abused adolescents.

Both these studies found that peer and family support, in combination with an individual's capacity to accept and utilize these resources, is critical for protecting individuals against poor mental health. Thus, these studies underline the importance of a close supporting surrounding environment, and the policy recommendations, therefore, suggest interventions to support and strengthen the parent, peer and child relations. Williams and Nelson-Gardell (2012) conclude that in order to promote resilience in sexually abused adolescents, interventions focusing on caregiver support and school engagement (proximal processes) or addressing caregiver education or economic assistance (contextual factors) will be the most effective and beneficial.

In summary, these studies give quite detailed guidance on (proximal) factors influencing the particular mental health outcomes in focus. However, given the weights on factors in the immediate, close environment, the recommendations that can be drawn from these studies focus mainly on interventions in the close and immediate environment, while somewhat downgrading actions are needed in the wider environment.

\section{Conclusion-different uses of Bronfenbrenner's theory; what is their value for guiding public mental health policy and practice?}

In summary, our study shows how the majority of mental health studies utilizing Bronfenbrenner's theory seem to use the early developed ecological system concepts without considering interactions within and between these systems. We do not believe that our review covers all studies within the field of public mental health 
that utilize Bronfenbrenner's theory. Still, it is striking that the vast majority of the identified articles use concepts of the theory in a way that was never intended by Bronfenbrenner himself. This finding supports Tudge et al.'s (2009) conclusion that one common misuse of early versions of Bronfenbrenner's theory is that it is used to map out contextual and individual factors contributing to an outcome while not analysing mutual interactions between the individual and the context, which was the explicit intention even with the initial version of the theory. Above, we claimed a "pragmatic view of theory", implying that concepts of a theory could be potentially useful (within a specific context) even if used in a way that was never intended. However, our results show that the recommendations for public mental health policy and practice that can be drawn from these studies are not very useful in that they are too broad and unspecific for suggesting what needs to be done for whom in order to influence a particular mental health outcome. As Stokols (1996, p. 288) puts it, "overly inclusive models are not likely to assist researchers in targeting selected variables for study, or clinicians and policy-makers in determining where, when, and how to intervene". Thus, we propose that using early concepts of Bronfenbrenner's theory without considering interactions within and between different ecological systems might be a less valuable use of the theory within the field of public mental health.

In contrast, our analysis shows that studies utilizing Bronfenbrenner's ecological system concepts, by clearly considering interactions within and between different ecological systems, can come up with most useful recommendations for public mental health promotion and interventions. These kinds of studies have the potential to rule out the "specific circumstances (e.g. intrapersonal, physical environmental, organizational, cultural) that account for the occurrence and prevalence of particular health problems, and a corresponding analysis of the contextual factors that are likely to influence the effectiveness of health-promotive interventions designed to reduce those problems" (Stokols 1996, p. 288). These kinds of recommendations may suggest what works for whom to prevent a particular mental health outcome. Therefore, we conclude that studies using early concepts of Bronfenbrenner's theory, by considering interactions within and between different ecological systems, can come up with valuable results for guiding public mental health interventions. This use of the theory offers a way to simultaneously focus on intrapersonal and environmental factors and the dynamic interplay between these factors in determining mental health. This way of using early concepts of the theory therefore corresponds very well to the ecological "needs" within public (mental) health for understanding the complexity of public health problems, including social inequality in health and the effects of place on health (McLaren and Hawe 2004). In addition, using concepts of Bronfenbrenner's theory in this way is well in line with a life course and social determinants of mental health perspective that emphasizes how mental health is shaped not only by individual factors but to a great extent by the social, economical and physical environments in which people live throughout their lives (WHO and Calouste Gulbenkian Foundation 2014).

We found only two mental health studies that had utilized the later concepts of proximal processes and the PPCT model of Bronfenbrenner's theory. This is despite the fact that these concepts were stated to be the most appropriate use of his theory 
(Bronfenbrenner and Evans 2000). The lack of studies utilizing these concepts might be due to the fact that this version of the theory is less known and spread in the scientific community, as indicated by Tudge et al. (2009). Alternatively, there may be a considered decision not to use these later concepts, given their main focus on proximal processes at the expense of environmental factors. Our analysis show that these final concepts do not obviously fit a public health and social determinants of mental health perspective, but might be more suitable within other fields such as psychotherapy where person-centred theories are the most appropriate to understand the structure and development of personality, taking into account dimensions of both temperament and character. The PPCT model is well in line with the ideas of Cloninger et al. (1993), who describe four dimensions of temperament: novelty seeking, harm avoidance, reward dependence, and persistence, which are independently heritable and manifest early in life. Cloninger et al. (1993) additionally describe three dimensions of character that mature in adulthood and influence personal and social effectiveness by insight learning about self-concepts. Selfconcepts vary according to the extent to which a person identifies the self as (1) an autonomous individual, (2) an integral part of humanity, and (3) an integral part of the universe as a whole. Consequently, our study suggests that within the field of public mental health research and practice, the later concepts of Bronfenbrenner's theory might not be the most useful. The final version of his theory, with its emphasis on proximal processes and the immediate environment, lacks a clear focus on how the social, economic and cultural environments that people are exposed to influence mental health. The policy implications that can be drawn from the PPCT model thus focus much more on the individual and consequently lean towards individual health promotion models, with an emphasis on changing individual health behaviour without considering the social and organizational context. These models have previously been extensively used in health promotion but have been criticized, not least for their "victim-blaming" ideology (McLeroy et al. 1988; Baum 2008). We should, however, remember that Bronfenbrenner was a developmental psychologist - a knowledge field with a clear focus on human growth and development in relation to age. Therefore, the latest concepts of Bronfenbrenner's theory could be seen as a return from a macro level perspective to a more individual-directed perspective where most developmental psychologists operate. In addition, one must also acknowledge that we were able to find very few articles that had tried to utilize these later, most mature concepts of Bronfenbrenner's theory. One of our two identified articles (Williams and Nelson-Gardell 2012) was also brought up in a recent re-evaluation of the uses of Bronfenbrenner's theory (Tudge et al. 2016) where it was evaluated as a study utilizing variables related to the PPCT, but without really testing the theory. The lack of illustrative examples of studies using the PPCT model limits a "fair" assessment of the value of using these concepts to guide public mental health interventions. Indeed, assessing the "influence of individual and contextual characteristics, through their influence on proximal processes" (Bronfenbrenner 1995), might be an appealing approach also in public mental health research. We believe that further development of an ecological approach in public mental health research would benefit from exploring proximal processes, operating on a more collective level, beyond Bronfenbrenner's 
more individually focused approach. Finally, even if we conclude that the PPCT model might not be the most useful version of Bronfenbrenner's theory within the field of public (mental) health, we do not claim that individual factors do not matter. In fact, equally important for public mental health policy and practice is to consider the variety of personal attributes such as psychological disposition and behavioural patterns that influence mental health (Stokols 1996). An ecological perspective that can "integrate the community wide, preventive strategies of public health and epidemiology with the individual-level, therapeutic and curative strategies of medicine" (Stokols 1996, p. 286) is needed within public mental health. This dual focus both on the surrounding environment and on personal attributes for explaining and promoting mental health can be achieved by utilizing early concepts of Bronfenbrenner's ecological theory, as long as interactions between and within ecological systems and individual factors are thoroughly investigated and considered.

Acknowledgements This research was financially supported by the Swedish Research Council Formas, dnr 259-2012-37.

Open Access This article is distributed under the terms of the Creative Commons Attribution 4.0 International License (http://creativecommons.org/licenses/by/4.0/), which permits unrestricted use, distribution, and reproduction in any medium, provided you give appropriate credit to the original author(s) and the source, provide a link to the Creative Commons license, and indicate if changes were made.

\section{References}

* Aston, H.J. 2014. An ecological model of mental health promotion for school communities: Adolescent views about mental health promotion in secondary schools in the UK. International Journal of Mental Health Promotion 16 (5): 289-307.

* Atzaba-Poria, N., A. Pike, and K. Deater-Deckard. 2004. Do risk factors for problem behavior act in a cumulative manner? An examination of ethnic minority and majority children through an ecological perspective. Journal of Child Psychology and Psychiatry 45 (4): 707-718.

Baum, F. 2008. The new public health. New York: Oxford University Press.

* Behnke, A.O., S.W. Plunkett, T. Sands, and M.Y. Bámaca-Colbert. 2011. The relationship between Latino adolescents perceptions of discrimination, neighborhood risk, and parenting on self-esteem and depressive symptoms. Journal of Cross-Cultural Psychology 42 (7): 1179-1197.

Bronfenbrenner, U. 1975. Reality and research in the ecology of human development. Proceedings of the American Philosophical Society 119 (6): 439-469.

Bronfenbrenner, U. 1977. Toward an experimental ecology of human development. American Psychologist 32 (7): 513-531.

Bronfenbrenner, U. 1978. The social role of the child in ecological perspective. Zeitschrift für Soziologie 7 (1): 4-20.

Bronfenbrenner, U. 1979. The ecology of human development: Experiments by nature and design. Cambridge: Harvard University Press.

Bronfenbrenner, U. 1986. Ecology of the family as a context for human development: Research perspectives. Developmental Psychology 22 (6): 723-742.

Bronfenbrenner, U., and S.J. Ceci. 1994. Nature-nurture reconceptualized in developmental perspective: A bioecological model. Psychological Review 101 (4): 568-586.

Bronfenbrenner, U. 1995. Developmental ecology through space and time: A future perspective. In Examining lives in context: Perspectives on the ecology of human development, ed. P. Moen, G.H. Elder Jr., and K. Lüscher, 619-647. Washington, DC: American Psychological Association. 
Bronfenbrenner, U., and G.W. Evans. 2000. Developmental science in the 21st century: Emerging questions, theoretical models, research designs and empirical findings. Social Development 9 (1): $115-125$.

Bryant, A. 2009. Grounded theory and pragmatism: The curious case of Anselm Strauss. Forum: Qualitative Social Research 10(3), Art. 2. http://nbn-resolving.de/urn:nbn:de:0114-fqs090325.

* Campbell, R., E. Dworkin, and Giannina Cabral. 2009. An ecological model of the impact of sexual assault on women's mental health. Trauma, Violence \& Abuse 10: 225-246.

Cloninger, C.R., D.M. Syrakic, and T.R. Przybeck. 1993. Psychobiological model of temperament and character. Archives of General Psychiatry 50 (12): 975-990.

* Grant, J., and P.B. Guerin. 2014. Applying ecological modelling to parenting for Australian refugee families. Journal of Transcultural Nursing 25: 325-333.

Grzywacz, J.P., and J. Fuqua. 2000. The social ecology of health: Leverage points and linkages. Behavioral Medicine 26 (3): 101-115.

* Hong, J.S., H. Cho, and A.-S. Lee. 2010. Revisiting the Virginia Tech shootings: An ecological systems analysis. Journal of Loss and Trauma: International Perspectives on Stress \& Coping 15 (6): 561-575.

* Hong, J.S., and D.L. Espelage. 2012. A review of research on bullying and peer victimization in school: An ecological system analysis. Aggression and Violent Behavior 17: 311-322.

* Huang, H., J.S. Hong, and D.L. Espelage. 2013. Understanding factors associated with bullying and peer victimization in Chinese schools within ecological contexts. Journal of Child and Family Studies 22: 881-892.

Jané-Llopis, E., M. Barry, C. Hosman, and V. Patel. 2005. Mental health promotion works: A review. Promotion \& Education 12 (9): 9-25.

Kendler, K.S. 2008. Explanatory models for psychiatric illness. American Journal of Psychiatry 165 (6): 695-702.

Kessler, R.C., et al. 2007. Lifetime prevalence and age-of-onset distributions of mental disorders in the World Health Organization's World Mental Health Survey Initiative. World Psychiatry 6 (3): 168-176.

Krieger, N. 2001. Theories for social epidemiology in the 21st century: An ecosocial perspective. International Journal of Epidemiology 30: 668-677.

Krieger, N. 2014. Got theory? On the 21st c. CE rise of explicit use of epidemiologic theories of disease distribution: A review and ecosocial analysis. Current Epidemiological Report 1: 45-56.

* Liem, J.H., K. Lustig, and C. Dillon. 2010. Depressive symptoms and life satisfaction among emerging adults: A comparison of high school dropouts and graduates. Journal of Adult Development 17: 33-43.

* McDaniel, B.T., S.M. Coyne, and E.K. Holmes. 2012. New mothers and media use: Associations between blogging, social networking, and maternal well-being. Maternal and Child Health Journal 16: $1509-1517$.

McLaren, L., and P. Hawe. 2004. Ecological perspectives in health research. Journal of Epidemiology and Community Health 59: 6-14.

McLeroy, K.R., D. Bibeau, A. Steckler, and K. Glanz. 1988. An ecological perspective on health promotion programs. Health Education Quarterly 15 (4): 351-377.

* Mutumba, M., and G.W. Harper. 2015. Mental health and support among young key populations: An ecological approach to understanding and intervention. Journal of the International AIDS Society 18 (S1): 19429.

* Pilgrim, N.A., and R. Blum. 2012. Adolescent mental and physical health in the English-speaking Caribbean. Review Panama Salud Publica 32 (1): 62-68.

Richard, L., L. Gauvin, and K. Raine. 2011. Ecological models revisited. Their uses and evolution in health promotion over two decades. Annual Review of Public Health 32: 307-326.

* Romano, E., L. Babchishin, R. Marquis, and S. Fréchette. 2015. Childhood maltreatment and educational outcomes. Trauma, Violence \& Abuse 16 (4): 418-437.

Rosa, E.M., and J. Tudge. 2013. Urie Bronfenbrenner's theory of human development: Its evolution from ecology to bioecology. Journal of Family Theory \& Review 5: 243-258.

Stokols, D. 1996. Translating social ecological theory into guidelines for community health promotion. American Journal of Health Promotion 10 (4): 282-298.

Sturgeon, S. 2007. Promoting mental health as an essential aspect of health promotion. Health promotion International 21 (S1): 36-41. 
Tudge, J.R.H., I. Mokrova, B.-E. Hatfield, and R.B. Karnik. 2009. Uses and misuses of Bronfenbrenner's bioecological theory of human development. Journal of Family Theory \& Review 1: 198-210.

Tudge, J.R.H., A. Payir, E. Mercon-Vargas, H. Cao, Y. Liang, J. Li, and L. O'Brien. 2016. Still misused after all these years? A reevaluation of the uses of Bronfenbrenner's bioecological theory of human development. Journal of Family Theory \& Review 8: 427-445.

* Upton Patton, D., J.S. Hong, A.B. Williams, and P. Allen-Meares. 2013. A review of research on school bullying among African American youth: An ecological systems analysis. Educational Psychology review 25: 245-260.

Whiteford, H.A., L. Degenhardt, J. Rehm, A.M. Baxter, A.J. Ferrari, H.E. Erskine, F.J. Charlson, R.E. Norman, A.D. Flaxman, N. Johns, R. Burstein, C.J.L. Murray, and T. Vos. 2013. Global burden of disease attributable to mental and substance use disorders: Findings from the Global Burden of Disease Study 2010. Lancet 382: 1575-1586.

* Williams, J., and D. Nelson-Gardell. 2012. Predicting resilience in sexually abused adolescents. Child Abuse and Neglect 36: 53-63.

World Health Organization (WHO). 1978. The Alma Ata Declaration. Geneva: WHO.

World Health Organization (WHO). 1986. The Ottawa Charter for health promotion. Geneva: WHO.

World Health Organization (WHO). 2005. Promoting mental health: Concepts, emerging evidence, practice. Geneva: WHO. http://www.who.int/mental_health/publications/promoting_mh_2005/en/. Accessed March 7, 2016.

World Health Organization (WHO). 2008. Closing the gap in a generation: Health equity through action on the social determinants of health. Geneva: WHO. Final Report of the Commission on Social Determinants of Health.

World Health Organization (WHO). 2012. Risks to mental health: An overview of vulnerabilities and risk factors. Background paper by WHO secretariat for the development of a comprehensive mental health action plan. Geneva: WHO http://www.who.int/mental_health/mhgap/risks_to_mental_ health_EN_27_08_12.pdf?ua=1. Accessed March 7, 2016.

World Health Organization (WHO). 2013. Mental health action plan 2013-2020. Geneva: WHO. http:// www.who.int/mental_health/publications/action_plan/en/. Accessed March 7, 2016.

World Health Organization (WHO). 2014. Mental health: Strengthening our response. Fact sheet No. 220. Geneva: WHO. http://www.who.int/mediacentre/factsheets/fs220/en/. Accessed March 7, 2016.

World Health Organization (WHO) and Calouste Foundation. 2014. Social determinants of mental health. Geneva: WHO.

* Yakushko, O., and K.M. Chronister. 2005. Immigrant women and counselling: The invisible others. Journal of Counselling \& Development 83: 292-298. 